\title{
A NOTE ON CANONICAL RICCI FORMS ON 2-STEP NILMANIFOLDS
}

\author{
LUIGI VEZZONI
}

(Communicated by Lei $\mathrm{Ni}$ )

\begin{abstract}
In this paper we prove that any left-invariant almost Hermitian structure on a 2-step nilmanifold is Ricci-flat with respect to the Chern connection and that it is Ricci-flat with respect to another canonical connection if and only if it is cosymplectic (i.e. $d^{*} \omega=0$ ).
\end{abstract}

\section{INTRODUCTION}

Let $(M, g, J, \omega)$ be an almost Hermitian manifold. Gauduchon introduced in [13] a 1-parameter family $\nabla^{t}$ of canonical Hermitian connections which can be distinguished by the properties of the torsion tensor $T$. In this family $\nabla^{1}$ corresponds to the so-called Chern connection, which can be defined as the unique Hermitian connection whose $(1,1)$-part of the torsion vanishes. In the quasi-Kähler case (i.e. when $\bar{\partial} \omega=0)$, the line $\left\{\nabla^{t}\right\}$ degenerates to a single point and the Chern connection is the unique canonical connection.

Any canonical connection $\nabla^{t}$ induces the so-called Ricci form $\rho^{t}(X, Y)=$ $2 \mathrm{itr} \omega R^{t}(X, Y)$, where $R^{t}$ denotes the curvature of $\nabla^{t}$. It turns out that $\rho^{t}$ is always a closed form which can be locally written as the derivative of the 1-form $\theta^{t}(X)=\sum_{r=1}^{n} g\left(\nabla_{X}^{t} Z_{r}, Z_{\bar{r}}\right)$, where $\left\{Z_{r}\right\}$ is a (local) unitary frame. Moreover, in the cosymplectic case (i.e. when $d \omega^{n-1}=0$ ), the line $\left\{\theta^{t}\right\}$ degenerates to a single point (see Corollary [3.3) and all the canonical connections have the same Ricci form.

The aim of this paper is to study the Ricci forms $\rho^{t}$ on 2-step nilmanifolds equipped with a left-invariant almost Hermitian structure. We recall that by definition a $k$-step nilmanifold is a compact quotient of a $k$-step nilpotent Lie group $G$ by lattice. Since we are considering left-invariant almost Hermitian structures, we can work on Lie algrebras in an algebraic fashion. Our main result is the following.

Theorem 1. Let $(\mathfrak{g}, g, J, \omega)$ be a 2-step nilpotent Lie algebra with an almost Hermitian structure. Then $(g, J)$ is Ricci-flat with respect to the Chern connection and it is Ricci-flat with respect to another canonical connection if and only if it is cosymplectic (i.e. $\left.d^{*} \omega=0\right)$.

Received by the editors June 8, 2011.

2000 Mathematics Subject Classification. Primary 53C15; Secondary 53B15.

The author was supported by the Project M.I.U.R. "Riemannian Metrics and Differentiable Manifolds" and by G.N.S.A.G.A. of I.N.d.A.M. 
This theorem has the following immediate consequence:

Corollary 1.1. Every left-invariant almost Hermitian structure on a nilmanifold associated to a 2-step Lie group is Ricci-flat with respect to the Chern connection.

\section{PRELIMINARIES ON CANONICAL CONNECTIONS}

Let $(M, g, J, \omega)$ be an almost Hermitian manifold, where $\omega$ is the fundamental form $\omega(\cdot, \cdot)=g(J \cdot, \cdot)$. The almost complex structure $J$ extends to $r$-forms as

$$
J \alpha\left(X_{1}, \ldots, X_{n}\right)=(-1)^{r} \alpha\left(J X_{1}, \ldots, J X_{n}\right),
$$

inducing the splittings

$$
T M \otimes \mathbb{C}=T^{1,0} M \oplus T^{0,1} M, \quad \Lambda^{r}(M, \mathbb{C})=\bigoplus_{p+q=r} \Lambda^{p, q} M,
$$

where $\Lambda^{r}(M, \mathbb{C})$ is the vector bundle of complex $r$-forms on $M$. In particular $\Lambda^{3} M$ splits as

$$
\Lambda^{3} M=\Lambda^{+} M \oplus \Lambda^{-} M,
$$

where $\Lambda^{+} M=\left(\Lambda^{2,1} M \oplus \Lambda^{1,2} M\right) \cap \Lambda^{3} M$ and $\Lambda^{-} M=\left(\Lambda^{3,0} M \oplus \Lambda^{0,3} M\right) \cap \Lambda^{3} M$. Given a 3-form $\gamma$ we denote by $\gamma^{+}$and $\gamma^{-}$the projections onto $\Lambda^{+} M$ and $\Lambda^{-} M$, respectively. Moreover, denoting by $\Omega^{2}(T M)$ the vector space of smooth sections of $\Lambda^{2} M \otimes T M$, we have the splitting

$$
\Omega^{2}(T M)=\Omega^{2,0}(T M) \oplus \Omega^{1,1}(T M) \oplus \Omega^{0,2}(T M),
$$

where

$$
\begin{aligned}
& \Omega^{2,0}(T M)=\left\{B \in \Omega^{2}(T M): B(J X, Y)=J B(X, Y)\right\} \\
& \Omega^{1,1}(T M)=\left\{B \in \Omega^{2}(T M): B(J X, J Y)=B(X, Y)\right\} \\
& \Omega^{0,2}(T M)=\left\{B \in \Omega^{2}(T M): B(J X, Y)=-J B(X, Y)\right\} .
\end{aligned}
$$

Hence any $B \in \Omega^{2}(T M)$ can be written as $B=B^{2,0}+B^{1,1}+B^{0,2}$. Notice that in terms of complex vector fields of type $(1,0)$ we have

$$
\begin{aligned}
B^{2,0}\left(Z_{i}, Z_{j}\right) & =B\left(Z_{i}, Z_{j}\right)+B\left(Z_{i}, Z_{j}\right)-\mathrm{i} J B\left(Z_{i}, Z_{j}\right)-\mathrm{i} J B\left(Z_{i}, Z_{j}\right) \\
& =2 B\left(Z_{i}, Z_{j}\right)-2 \mathrm{i} J B\left(Z_{i}, Z_{j}\right) .
\end{aligned}
$$

In particular the condition $B^{2,0}=0$ can be written in terms of $(1,0)$ vector fields as $B\left(Z_{i}, Z_{j}\right) \in T^{0,1} M$. Furthermore $\Omega^{2}(T M)$ splits as

$$
\Omega^{2}(T M)=\Omega_{b}^{2}(T M) \oplus \Omega_{c}^{2}(T M),
$$

where

$$
\begin{aligned}
& g\left(B_{b}(X, Y), Z\right)=\frac{1}{2}(g(B(X, Y), Z)-g(B(Z, X), Y)-g(B(Y, Z), X)), \\
& g\left(B_{c}(X, Y), Z\right)=\frac{1}{2}(g(B(X, Y), Z)+g(B(Z, X), Y)+g(B(Y, Z), X)) .
\end{aligned}
$$

Now we consider connections on $M$. A connection $\nabla$ on $M$ is called Hermitian if $\nabla J=0, \nabla g=0$. It is well known that every almost Hermitian manifold admits 
Hermitian connections. We denote by $\mathcal{C}$ the space of Hermitian connections on $M$. Gauduchon introduced in 13 the following special class of Hermitian connections:

Definition 2.1. A connection $\nabla \in \mathcal{C}$ is called canonical if its torsion $T$ satisfies $T_{b}^{1,1}=0$.

From [13] it follows that any canonical connection $\nabla$ can be written as

$$
\begin{aligned}
g\left(\nabla_{X} Y, Z\right)= & g\left(D_{X} Y, Z\right)+\frac{t-1}{4}\left(d^{c} \omega\right)^{+}(X, Y, Z)+\frac{t+1}{4}\left(d^{c} \omega\right)^{+}(X, J Y, J Z) \\
& -g(X, N(Y, Z))+\frac{1}{2}\left(d^{c} \omega\right)^{-}(X, Y, Z)
\end{aligned}
$$

for some $t \in \mathbb{R}$, where $d^{c}$ is the operator acting on $r$-forms as $d^{c}=(-1)^{r} J d J$ and $N$ denotes the Nijenhuis tensor $N(X, Y)=[J X, J Y]-[X, Y]-J([J X, Y]+[X, J Y])$.

For $t \in \mathbb{R}$ we denote by $\nabla^{t}$ the corresponding canonical connection. In the special case of a quasi-Kähler structure (i.e. $\bar{\partial} \omega=0$ ) the space of canonical connections reduces to a single point, while if $J$ is integrable (i.e. $N=0$ ) equation (2.1) reduces to

$$
g\left(\nabla_{X}^{t} Y, Z\right)=g\left(D_{X} Y, Z\right)+\frac{t-1}{4}\left(d^{c} \omega\right)(X, Y, Z)+\frac{t+1}{4}\left(d^{c} \omega\right)(X, J Y, J Z) .
$$

For the parameters $t=1,0,-1$, the family (2.1) gives the following remarkable cases:

- $t=1$. In this case $\nabla^{1}$ is called the Chern connection. This connection can be defined as the unique Hermitian connection satisfying $T^{1,1}=0$.

- $t=0$. In this case $\nabla^{0}$ is called the first canonical connection. This connection can be defined as the unique Hermitian connection whose torsion satisfies $T^{2,0}=0$.

- $t=-1$. In this case the connection $\nabla^{-1}$ is important in the complex case where it is known as the Bismut connection. Indeed, if $J$ is integrable, then $\nabla^{-1}$ can be defined as the unique Hermitian connection having totally skew-symmetric torsion (see [6]).

\section{CANONicAl RicCi FORMS}

Let $\left(M^{2 n}, g, J\right)$ be an almost Hermitian manifold and let $\mathcal{C}$ be the space of the associated Hermitian connections. For any $\nabla \in \mathcal{C}$ the Ricci form is defined as $\rho(X, Y)=2 \operatorname{itr}_{\omega} R(X, Y)$, where $R$ is the curvature tensor $R(X, Y):=\left[\nabla_{X}, \nabla_{Y}\right]-$ $\nabla_{[X, Y]}$. Such a form is always closed and locally it satisfies $\rho=d \theta$, where $\theta(X)=$ $\sum_{r=1}^{n} g\left(\nabla_{X} Z_{r}, Z_{\bar{r}}\right)$ and $\left\{Z_{r}\right\}$ is a local unitary frame. In the case of a canonical connection $\nabla^{t} \in \mathcal{C}$ we use the notation $\rho^{t}$ and $\theta^{t}$. We denote by the natural isomorphism between vector fields and 1-forms induced by $g$. Namely, if $X$ is a vector field, then we denote by $X^{\natural}$ the 1 -form $X^{\natural}(Y)=g(X, Y)$. We have the following.

Proposition 3.1. $\theta^{t}$ is locally defined by

$$
\theta^{t}(X)=\sum_{r=1}^{n} \mathrm{i} \Im \mathfrak{m}\left\{g\left(\left[X+t \mathrm{i} J X, Z_{r}\right], Z_{\bar{r}}\right)\right\}+\frac{1}{2} \mathrm{i}(t-1) g\left(d^{*} \omega, X^{\natural}\right)
$$

for any vector field $X$. 
Proof. First of all we note that if $Z_{r}$ is a vector field of type $(1,0)$, then

$$
\begin{gathered}
N\left(Z_{r}, Z_{\bar{r}}\right)=\left(d^{c} \omega\right)^{-}\left(X, Z_{r}, Z_{\bar{r}}\right)=0 \\
\left(d^{c} \omega\right)^{+}\left(X, Z_{r}, Z_{\bar{r}}\right)=d^{c} \omega\left(X, Z_{r}, Z_{\bar{r}}\right)=-d \omega\left(J X, Z_{r}, Z_{\bar{r}}\right) .
\end{gathered}
$$

Hence if $\left\{Z_{r}\right\}$ is a local unitary frame, using equation (2.1) we get

$$
\begin{aligned}
\theta^{t}(X) & =\sum_{r=1}^{n}\left\{g\left(D_{X} Z_{r}, Z_{\bar{r}}\right)+\frac{t-1}{4}\left(d^{c} \omega\right)\left(X, Z_{r}, Z_{\bar{r}}\right)+\frac{t+1}{4}\left(d^{c} \omega\right)\left(X, J Z_{r}, J Z_{\bar{r}}\right)\right\} \\
& =\sum_{r=1}^{n}\left\{g\left(D_{X} Z_{r}, Z_{\bar{r}}\right)-\frac{t}{2} d \omega\left(J X, Z_{r}, Z_{\bar{r}}\right)\right\} .
\end{aligned}
$$

Now

$$
\begin{aligned}
2 g\left(D_{X} Z_{r}, Z_{\bar{r}}\right)= & X g\left(Z_{r}, Z_{\bar{r}}\right)-Z_{\bar{r}} g\left(X, Z_{r}\right)+Z_{r} g\left(X, Z_{\bar{r}}\right)+g\left(\left[X, Z_{r}\right], Z_{\bar{r}}\right) \\
& +g\left(\left[Z_{\bar{r}}, X\right], Z_{r}\right)-g\left(\left[Z_{r}, Z_{\bar{r}}\right], X\right) \\
= & -Z_{\bar{r}} g\left(X, Z_{r}\right)+Z_{r} g\left(X, Z_{\bar{r}}\right)+g\left(\left[X, Z_{r}\right], Z_{\bar{r}}\right)+g\left(\left[Z_{\bar{r}}, X\right], Z_{r}\right) \\
& -g\left(\left[Z_{r}, Z_{\bar{r}}\right], X\right)
\end{aligned}
$$

and

$$
\begin{aligned}
d \omega\left(J X, Z_{r}, Z_{\bar{r}}\right)= & (J X) \omega\left(Z_{r}, Z_{\bar{r}}\right)-Z_{r} \omega\left(J X, Z_{\bar{r}}\right)+Z_{\bar{r}} \omega\left(J X, Z_{r}\right) \\
& -\omega\left(\left[J X, Z_{r}\right], Z_{\bar{r}}\right)-\omega\left(\left[Z_{\bar{r}}, J X\right], Z_{r}\right)-\omega\left(\left[Z_{r}, Z_{\bar{r}}\right], J X\right) \\
= & Z_{r} g\left(X, Z_{\bar{r}}\right)-Z_{\bar{r}} g\left(X, Z_{r}\right)+\mathrm{i} g\left(\left[J X, Z_{r}\right], Z_{\bar{r}}\right)+\mathrm{i} g\left(\left[Z_{\bar{r}}, J X\right], Z_{r}\right) \\
& -g\left(\left[Z_{r}, Z_{\bar{r}}\right], X\right) .
\end{aligned}
$$

Then we have

$$
\begin{aligned}
\theta^{t}(X)=\frac{1}{2} \sum_{r=1}^{n}\{g([X+ & \left.\left.t \mathrm{i} J X, Z_{r}\right], Z_{\bar{r}}\right)-g\left(\left[X-t \mathrm{i} J X, Z_{\bar{r}}\right], Z_{r}\right)+g\left(\left[Z_{r}, Z_{\bar{r}}\right], t X-X\right) \\
+ & \left.(1-t) Z_{r} g\left(X, Z_{\bar{r}}\right)-(1-t) Z_{\bar{r}} g\left(X, Z_{r}\right)\right\} \\
=\sum_{r=1}^{n}\{\mathrm{i} \Im \mathfrak{m}\{ & \left.g\left(\left[X+t \mathrm{i} J X, Z_{r}\right], Z_{\bar{r}}\right)+(1-t) Z_{r} g\left(X, Z_{\bar{r}}\right)\right\} \\
- & \left.\frac{1}{2}(1-t) g\left(\left[Z_{r}, Z_{\bar{r}}\right], X\right)\right\}
\end{aligned}
$$

So in order to prove the statement we have to show that

$$
\sum_{r=1}^{n}\left\{\Im \mathfrak{m}\left\{Z_{r} g\left(X, Z_{\bar{r}}\right)\right\}+\mathrm{i} \frac{1}{2} g\left(\left[Z_{r}, Z_{\bar{r}}\right], X\right)\right\}=-\frac{1}{2} g\left(\omega, d X^{\natural}\right) .
$$


We can write $X=\sum_{r=1}^{n}\left(X_{r} Z_{r}+X_{\bar{r}} Z_{\bar{r}}\right)$ and $X^{\natural}=\sum_{r=1}^{n}\left(X_{r} \zeta^{r}+X_{\bar{r}} \zeta^{\bar{r}}\right)$, where $\left\{\zeta^{r}\right\}$ is the coframe dual to $\left\{Z_{r}\right\}$. Then we get

$$
\begin{aligned}
g\left(\omega, d X^{\natural}\right) & =\mathrm{i} \sum_{k=1}^{n} g\left(\zeta^{k} \wedge \zeta^{\bar{k}}, d X^{\natural}\right) \\
& =\mathrm{i} \sum_{k, r=1}^{n}\left(Z_{r}\left(X_{\bar{r}}\right)-Z_{\bar{r}}\left(X_{r}\right)-X^{\natural}\left(\left[Z_{r}, Z_{\bar{r}}\right]\right)\right) g\left(\zeta^{k} \wedge \zeta^{\bar{k}}, \zeta^{r} \wedge \zeta^{\bar{r}}\right) \\
& \left.=\mathrm{i} \sum_{k=1}^{n} Z_{k}\left(X_{\bar{k}}\right)-Z_{\bar{k}}\left(X_{k}\right)-X^{\natural}\left(\left[Z_{k}, Z_{\bar{k}}\right]\right)\right) \\
& =-2 \sum_{k=1}^{n} \Im \mathfrak{m}\left\{Z_{k}\left(X_{\bar{k}}\right)\right\}-\mathrm{i} \sum_{k, s=1}^{n}\left(B_{k \bar{k}}^{s} X_{s}+B_{k \bar{k}}^{\bar{s}} X_{\bar{s}}\right) \\
& =-\sum_{k=1}^{n}\left(2 \Im \mathfrak{m}\left\{Z_{k}\left(X_{\bar{k}}\right)\right\}+\mathrm{i} g\left(\left[Z_{k}, Z_{\bar{k}}\right], X\right)\right),
\end{aligned}
$$

where with $B$ we denote the components of the brackets.

Corollary 3.2. The following formulae hold:

- $\theta^{1}(X)=2 \mathrm{i} \sum_{r=1}^{n} \Im \mathfrak{m} g\left(\left[X^{0,1}, Z_{r}\right], Z_{\bar{r}}\right)$;

- $\theta^{0}(X)=\mathrm{i} \sum_{r=1}^{n} \Im \mathfrak{m}\left\{g\left(\left[X, Z_{r}\right], Z_{\bar{r}}\right)\right\}-\mathrm{i} \frac{1}{2} g\left(d^{*} \omega, X^{\natural}\right)$;

- $\theta^{-1}(X)=2 \mathrm{i} \sum_{r=1}^{n} \Im \mathfrak{m}\left\{g\left(\left[X^{1,0}, Z_{r}\right], Z_{\bar{r}}\right)\right\}-\mathrm{i} g\left(d^{*} \omega, X^{\natural}\right)$.

It is useful to write formula (3.1) in real coordinates. In order to do this we write $Z_{r}=\frac{1}{\sqrt{2}}\left(e_{r}-\mathrm{i} J e_{r}\right)$ for a suitable orthonormal frame $\left\{e_{1}, \ldots, e_{n}, J e_{1}, \ldots, J e_{n}\right\}$. Then a direct computation gives

$$
\begin{aligned}
2 \Im \mathfrak{m} & \left\{g\left(\left[X+t \mathrm{i} J X, Z_{r}\right], Z_{\bar{r}}\right)\right\}=\Im \mathfrak{m}\left\{g\left(\left[X+t \mathrm{i} J X, e_{r}-\mathrm{i} J e_{r}\right], e_{r}+\mathrm{i} J e_{r}\right)\right\} \\
= & g\left(\left[X, e_{r}\right], J e_{r}\right)-g\left(\left[X, J e_{r}\right], e_{r}\right)+\operatorname{tg}\left(\left[J X, e_{r}\right], e_{r}\right)+\operatorname{tg}\left(\left[J X, J e_{r}\right], J e_{r}\right)
\end{aligned}
$$

and

$$
\begin{aligned}
\theta^{t}(X)= & \frac{1}{2} \mathrm{i} \sum_{r=1}^{n}\left\{g\left(\left[X, e_{r}\right], J e_{r}\right)-g\left(\left[X, J e_{r}\right], e_{r}\right)+\operatorname{tg}\left(\left[J X, e_{r}\right], e_{r}\right)\right. \\
& \left.+\operatorname{tg}\left(\left[J X, J e_{r}\right], J e_{r}\right)\right\} \\
& +\frac{1}{2} \mathrm{i}(t-1) g\left(d^{*} \omega, X^{\natural}\right) .
\end{aligned}
$$

A remarkable consequence of formula (3.1) is the following.

Corollary 3.3. All canonical connections of a cosymplectic structure have the same Ricci form.

Proof. It is enough to show that $\theta^{1}=\theta^{-1}$. Since the cosymplectic condition $d^{*} \omega=$ 0 implies

$$
\theta^{-1}(X)=\sum_{r=1}^{n} 2 \mathrm{i} \Im \mathfrak{m}\left\{g\left(\left[X^{1,0}, Z_{r}\right], Z_{\bar{r}}\right)\right\}
$$


we have

$$
\begin{aligned}
\theta^{-1}(X) & =-\sum_{r=1}^{n} 2 \mathrm{i} \Im \mathfrak{m}\left\{g\left(\left[X^{0,1}, Z_{\bar{r}}\right], Z_{r}\right)\right\} \\
& =-\sum_{r=1}^{n} 2 \mathrm{i} \Im \mathfrak{m}\left\{g\left(D_{X^{0,1}} Z_{\bar{r}}, Z_{r}\right)-g\left(D_{Z_{\bar{r}}} X^{0,1}, Z_{r}\right)\right\} \\
& =\sum_{r=1}^{n} 2 \mathrm{i} \Im \mathfrak{m}\left\{g\left(D_{X^{0,1}} Z_{r}, Z_{\bar{r}}\right)-g\left(D_{Z_{\bar{r}}} X^{0,1}, Z_{r}\right)\right\} \\
& =\theta^{1}(X)+\sum_{r=1}^{n} 2 \mathrm{i} \Im \mathfrak{m}\left\{g\left(D_{Z_{r}} X^{0,1}, Z_{\bar{r}}\right)-g\left(D_{Z_{\bar{r}}} X^{0,1}, Z_{r}\right)\right\}
\end{aligned}
$$

Now we observe that $\sum g\left(D_{Z_{r}} X^{0,1}, Z_{\bar{r}}\right)=-\sum g\left(X^{0,1}, D_{Z_{r}} Z_{\bar{r}}\right)=0$, since the cosymplectic condition forces $\sum D_{Z_{\bar{r}}} Z_{r}$ to be of type $(1,0)$ (see e.g. [16]). The last step consists of showing that $\sum \Im \mathfrak{m}\left\{g\left(D_{Z_{\bar{r}}} X^{0,1}, Z_{r}\right)\right\}=0$. Here it is enough to consider the identity

$$
\sum_{r=1}^{n}\left\{\Im \mathfrak{m}\left\{Z_{r} g\left(X, Z_{\bar{r}}\right)\right\}+\mathrm{i} \frac{1}{2} g\left(\left[Z_{r}, Z_{\bar{r}}\right], X\right)\right\}=\sum_{r=1}^{n} \Im \mathfrak{m}\left\{g\left(D_{Z_{\bar{r}}} X^{0,1}, Z_{r}\right)\right\},
$$

which can be checked by performing a direct computation. Then equation (3.2) implies the statement.

Remark 3.4. In the Hermitian case this last result was already known. In fact, it can be deduced from formula (8) of [14]. Another proof of this fact can be found in $[17$.

\section{CAnonical Ricci forms on Lie Algebras}

Now we restrict our attention to left-invariant almost Hermitian structures on Lie groups (or more generally to left-invariant almost Hermitian structures on quotients of Lie groups by lattices). Since here all the computations are purely algebraic, we may assume to work on a Lie algebra $(\mathfrak{g},[\cdot, \cdot])$ equipped with an almost Hermitian structure $(g, J)$. An almost Hermitian structure on a Lie algebra is a pair $(g, J)$, where $J$ is an endomorphism of $\mathfrak{g}$ satisfying $J^{2}=-\mathrm{Id}$ and $g$ is a $J$-Hermitian inner product. The bracket of $\mathfrak{g}$ has no a priori relation with $J$. The pair $(g, J)$ induces as usual the fundamental form $\omega(\cdot, \cdot)=g(J \cdot, \cdot)$.

Proposition 3.1 implies the following.

Proposition 4.1. Let $(\mathfrak{g},[\cdot, \cdot], g, J)$ be a Lie algebra with an almost Hermitian structure. For any $t \in \mathbb{R}$ the following formula holds:

$$
\theta^{t}(X)=\frac{1}{2} \mathrm{i}\left\{-\operatorname{tr}\left(\operatorname{ad}_{X} \circ J\right)+t \operatorname{tr} \operatorname{ad}_{J X}+(t-1) g\left(d^{*} \omega, X^{\natural}\right)\right\} .
$$

Moreover if $(\mathfrak{g},[\cdot, \cdot])$ is unimodular $\left(\right.$ i.e. $\operatorname{tr} \operatorname{ad}_{X}=0$ for any $\left.X \in \mathfrak{g}\right)$, then

$$
\rho^{t}(X, Y)=\frac{1}{2} \mathrm{i} \operatorname{tr}\left(\operatorname{ad}_{[X, Y]} \circ J\right)-\frac{1}{2} \mathrm{i}(t-1) g\left(d^{*} \omega,[X, Y]^{\natural}\right)
$$

and $(g, J)$ is Ricci-flat with respect to any canonical connection if and only if it is cosymplectic. 
Proof. The only nontrivial part of the statement is the last assertion. So we have just to show that condition $g\left(\omega, d[X, Y]^{\natural}\right)=0$ is equivalent to $d^{*} \omega=0$. We can write $\mathfrak{g}=[\mathfrak{g}, \mathfrak{g}] \oplus[\mathfrak{g}, \mathfrak{g}]^{\perp}$. Let $X \in[\mathfrak{g}, \mathfrak{g}]^{\perp}$. Then

$$
d X^{\natural}(Z, W)=-g(X,[Z, W])=0 .
$$

Hence for every $X \in[\mathfrak{g}, \mathfrak{g}]^{\perp}, d X^{\natural}=0$. This implies that $d \mathfrak{g}^{*}=d\left([\mathfrak{g}, \mathfrak{g}]^{\natural}\right)$ and the claim follows.

An interesting consequence of this last proposition is the following.

Now we can prove Theorem 1

Proof of Theorem 1, Let $(\mathfrak{g},[\cdot, \cdot], g, J)$ be a 2-step nilpotent Lie algebra with an almost Hermitian structure. Then, taking into account that $\mathfrak{g}$ is unimodular, the 2step condition implies that $[\mathfrak{g}, \mathfrak{g}]$ is contained in the center of $\mathfrak{g}$ and $\operatorname{tr}\left(\operatorname{ad}_{[X, Y]}{ }^{\circ} J\right)=0$ for every $X, Y \in \mathfrak{g}$. Then formula (4.2) reduces to

$$
\rho^{t}(X, Y)=\frac{1}{2} \mathrm{i}(1-t) g\left(d^{*} \omega,[X, Y]^{\natural}\right),
$$

and the first claim follows.

On the other hand, assume we have a 2-step nilpotent Lie algebra $\mathfrak{g}$ equipped with an almost Hermitian structure $(g, J)$ such that $J$ preserves the center of $\mathfrak{g}$. Then taking into account that

$$
\operatorname{tr}\left(\operatorname{ad}_{[X, Y]} \circ J\right)=0, \quad \operatorname{ad}_{J[X, Y]}=0
$$

for every $X, Y \in \mathfrak{g}$, we have that also in this case formula (4.2) reduces to (4.3) and the second claim follows.

Proposition 4.1 allows us to describe the behavior of $\left\{\rho^{t}\right\}$ for some special almost Hermitian structures:

Proposition 4.2. Let $(\mathfrak{g}[\cdot, \cdot], g, J)$ be an almost Hermitian Lie algebra.

- If $J$ is bi-invariant $($ i.e. $[J \cdot, \cdot]=J[\cdot, \cdot])$, then

$$
\theta^{t}(X)=(t-1) \mathrm{i} \operatorname{tr}\left(\operatorname{ad}_{J X}\right), \quad \rho^{t}(X, Y)=\mathrm{i}(1-t) \operatorname{tr}\left(\operatorname{ad}_{[J X, Y]}\right) .
$$

- If $J$ is anti-bi-invariant (i.e. $[J \cdot, \cdot]=-J[\cdot, \cdot])$, then

$$
\theta^{t}=0, \quad \rho^{t}=0 .
$$

- If $J$ is abelian (i.e. $[J \cdot, J \cdot]=[\cdot, \cdot])$, then

$$
\begin{aligned}
& \theta^{t}(X)=\frac{1}{2} \mathrm{i}\left\{(1+t) \operatorname{tr}\left(\operatorname{ad}_{J X}\right)+(t-1) g\left(d^{*} \omega, X^{\natural}\right)\right\}, \\
& \rho^{t}(X)=\frac{1}{2} \mathrm{i}\left\{-(1+t) \operatorname{tr}\left(\operatorname{ad}_{J[X, Y]}\right)+(1-t) g\left(d^{*} \omega,[X, Y]^{\natural}\right)\right\} .
\end{aligned}
$$

- If $J$ is anti-abelian (i.e. $[J \cdot, J \cdot]=-[\cdot, \cdot])$, then

$$
\theta^{t}(X)=\frac{1}{2} \mathrm{i}(1+t) \operatorname{tr}\left(\operatorname{ad}_{J X}\right), \quad \rho^{t}(X, Y)=-\frac{1}{2} \mathrm{i}(1+t) \operatorname{tr}\left(\operatorname{ad}_{J[X, Y]}\right) .
$$

In particular in the unimodular case bi-invariant, anti-bi-invariant and anti-abelian almost Hermitian structures are Ricci-flat with respect to any canonical connection, while in the abelian case, $\rho^{t}$ is given by the following formula:

$$
\rho^{t}(X, Y)=\frac{1}{2} \mathrm{i}(1-t) g\left(d^{*} \omega,[X, Y]^{\natural}\right),
$$

and $\rho^{t}=0$ for $t \neq 0$ if and only if $(g, J)$ is a cosymplectic structure. 
Remark 4.3. We note the following facts:

- The bi-invariant condition $[J \cdot, \cdot]=J[\cdot, \cdot]$ is equivalent to requiring that the simply connected Lie group associated to $(\mathfrak{g}, J)$ is a complex Lie group. The fact that a bi-invariant almost Hermitian structure on a unimodular Lie algebra is Ricci-flat with respect any canonical connection has already been proved by Grantcharov in [15.

- The anti-bi-invariant condition $[J \cdot, \cdot]=-J[\cdot, \cdot]$ is equivalent to requiring that any $J$-compatible inner product on $\mathfrak{g}$ is quasi-Kähler and flat with respect to the Chern connection $\nabla^{1}$ (see [9]).

- The abelian condition $[J \cdot, J \cdot]=[\cdot, \cdot]$ was introduced in [4] and was intensely studied in [3, 5, 12, 8, 18. This condition is equivalent to requiring that $\mathfrak{g}^{1,0}$ is an abelian Lie algebra.

- Finally, the anti-abelian condition $[J \cdot, J \cdot]=-[\cdot, \cdot]$ was studied in [11].

Remark 4.4. Theorem 1 can be applied to the Heisenberg Lie algebras $\mathfrak{h}_{n}(\mathbb{R})$ and $\mathfrak{h}_{n}(\mathbb{C})$. That is in accord with Theorem 4.1 of $[20$ ] and Proposition 4.10 and 4.11 of [10]. Moreover things work differently either in the 3-step nilpotent case or in the 2-step solvable case (see [10]).

\section{ACKNOWLEDGMENTS}

The research of this paper was motivated by a conversation with Simon Salamon. The author is very grateful to him. Furthermore, the author is grateful to Gueo Grantcharov for useful conversations and remarks and to Nicola Enrietti for an important observation on the presentation of the main result.

\section{REFERENCES}

[1] Abbena E.: An example of an almost Kähler manifold which is not Kählerian. Boll. Un. Mat. Ital. $A$ (6) 3 (1984), no. 3, 383-392. MR769169 (86a:53036)

[2] Abbena E., Garbiero S., Salamon S.: Almost Hermitian geometry on six dimensional nilmanifolds. Ann. Scuola Norm. Sup. Pisa Cl. Sci. (4) 30 (2001), no. 1, pp. 147-170. MR1882028 (2003b:53028)

[3] Andrada A., Barberis M.L., Dotti I.G.: Classification of abelian complex structures on 6dimensional Lie algebras. J. London Math. Soc. (2) 83 (2011), no. 1, 232-255. MR2763953

[4] Barberis M. L., Dotti Miatello Isabel G., Miatello Roberto J.: On certain locally homogeneous Clifford manifolds. Ann. Global Anal. Geom. 13 (1995), no. 3, 289-301. MR1344484 (96g:53060)

[5] Barberis M. L., Dotti I. and Verbitsky M.: Canonical bundles of complex nilmanifolds, with applications to hypercomplex geometry, Math. Research Letters 16 (2) (2009), 331-347. MR2496748 (2010g:53083)

[6] Bismut J. M.: A local index theorem for non-Kähler manifolds, Mathematische Annalen 284 (1989), no. 4, 681-699. MR1006380 (91i:58140)

[7] Chern S.-S.: Characteristic classes of Hermitian manifolds, Ann. of Math. (2) 47 (1946), 85-121. MR0015793 (7:470b)

[8] Console S., Fino A., Poon Y. S.: Stability of abelian complex structures. Internat. J. Math. 17 (2006), no. 4, 401-416. MR2220652(2007f:32013)

[9] Di Scala A.J., Vezzoni L.: Quasi-Kähler manifolds with trivial Chern holonomy (2007). To appear in Math Z., arXiv:0807.1664.

[10] Di Scala J.A., Vezzoni L.: Chern-flat and Ricci-flat invariant almost Hermitian structures. Ann. Global Anal. Geom. 40 (2011), no. 1, 21-45. MR.2795448

[11] Di Scala A.J., Lauret J., Vezzoni L.: Quasi-Kähler Chern-flat manifolds and complex 2-step nilpotent Lie algebras. To appear in Ann. Sc. Norm. Super. Pisa Cl. Sci., arXiv:0911.5655 
[12] Dotti Isabel G., Fino A.: Hypercomplex nilpotent Lie groups. Global differential geometry: the mathematical legacy of Alfred Gray (Bilbao, 2000), 310-314, Contemp. Math., 288, Amer. Math. Soc., Providence, RI, 2001. MR 1871024 (2002j:53050)

[13] Gauduchon P.: Hermitian connections and Dirac operators. Boll. Un. Mat. Ital. B (7) 11 (1997), no. 2, suppl., 257-288. MR.1456265 (98c:53034)

[14] Grantcharov D., Grantcharov G., Poon, Y. S.: Calabi-Yau connections with torsion on toric bundles. J. Differential Geom. 78 (2008), no. 1, 13-32. MR2406264 (2009g:32052)

[15] Grantcharov G.: Geometry of compact complex homogeneous spaces with vanishing first Chern class. Adv. in Math. 226, no. 4 (2011), 3136-3159. MR2764884

[16] Gudmundsson S., Wood J. C.: Harmonic morphisms between almost Hermitian manifolds. Boll. Un. Mat. Ital. B (7) 11 (1997), no. 2, suppl., 185-197. MR1456260 (98i:58069)

[17] Liu K., Yang X.: Geometry of Hermitian manifolds. arXiv:1011.0207.

[18] Maclaughlin C., Pedersen H., Poon, Y. S., Salamon S.: Deformation of 2-step nilmanifolds with abelian complex structures. J. London Math. Soc. (2) 73 (2006), no. 1, 173-193. MR2197377 (2007b:32020)

[19] Salamon S.: Riemannian geometry and holonomy groups. Pitman Research Notes in Mathematics Series, 201. Longman Scientific \& Technical, Harlow; copublished in the United States with John Wiley \& Sons, Inc., New York, 1989. MR1004008 (90g:53058)

[20] Tosatti V., Weinkove B: The Calabi-Yau equation on the Kodaira-Thurston manifold, J. Inst. Math. Jussieu 10 (2011), no.2, 437-447. MR2787695

Dipartimento di Matematica, Università di Torino, Torino, Italy

E-mail address: luigi.vezzoni@unito.it 